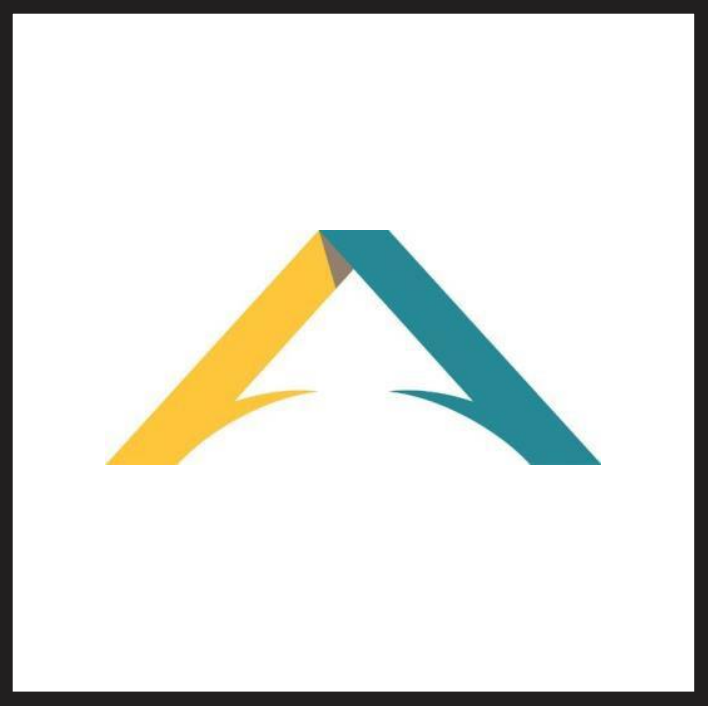

Revista

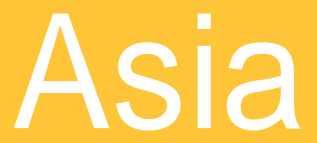

América

Latina

ISSN 2524-9347

Grupo de Estudios sobre Asia y América Latina Instituto de Estudios de América Latina y el Caribe Universidad de Buenos Aires

(c)

EU

DE BA 


\section{LA DIFÍCIL TAREA DE LLEVAR LA DEMOCRACIA A ASIA: UNA ENTREVISTA A ALBERTINA PITERBARG}

\section{THE DIFFICULT TASK OF INTRODUCING DEMOCRACY IN ASIA: AN INTERVIEW WITH ALBERTINA PITERBARG}

En el año 2008, la experta electoral Albertina Piterbarg se mudó junto con su familia a Timor Oriental para trabajar en la misión de Cascos Azules de Naciones Unidas. Este pequeño país, ubicado en el extremo meridional del archipiélago indonesio y en la parte oriental de la isla de Timor, es una de las naciones más jóvenes del mundo. Tras siglos como colonia portuguesa, seguidos por años de anexión forzosa a Indonesia, los timorenses alcanzaron la independencia en 2002.

Timor Oriental emergió como nación independiente siendo el país más pobre de Asia y con una sociedad devastada por la violencia que marcó la retirada indonesia. Naciones Unidas intervino en el proceso de transición, haciéndose cargo de todas las funciones de gobierno y la pacificación del territorio hasta que un gobierno civil pudiera hacerse cargo. En 2006, el retorno de la violencia llevó al establecimiento de la Misión Integrada de las Naciones Unidas en Timor Oriental (o UNMIT por sus siglas en inglés) para el restablecimiento y mantenimiento de la paz.

Fue en el contexto de esta misión, que Albertina llegó para trabajar en la preparación de las primeras elecciones de sucos (aldeas) de 2009. En sus cuatro años en el país, trabajó junto a múltiples actores involucrados en la preparación, realización y supervisión de procesos electorales como funcionarios, organizaciones de la sociedad civil, organizaciones internacionales, iglesias y jefes tribales. Charlamos con ella sobre su experiencia profesional y personal en un país tan remoto y en proceso de crear sus instituciones democráticas.

\section{¿Cómo fue la vida en Timor Oriental? Vos fuiste con tu familia, con dos hijas.}

Maravillosa, fue un verano dorado. Fueron cuatro años de vivir en un lugar increíble, dificilísimo porque fue muy jugado lo nuestro ya que no hay salud ni agua potable y todo eso. Pero fue una experiencia genial, porque es una sociedad a la que le encanta los chicos, porque los chicos son riqueza, los chicos son alegría. Entonces para ellos, para mis colegas de la comisión electoral, que yo haya ido con mis hijas era una muestra de respeto. Aunque me preguntaban: 
“¿cuántas hijas tiene usted?”, y cuando les decía que tenía dos, ellos me decían: “¡No, más más más! Más tiene que traer”. Dos hijas para ellos es nada. Pero bueno, el hecho de que yo estaba con mis hijas y mis hijas estaban ahí era una muestra de mi respeto hacia ellos y para ellos yo era respetable por estar con mis hijas. O sea, para ellos, alguien que no tiene hijos no es bien visto.

En largo plazo esto debe ser todo un problema demográfico. En especial, en un país con poco territorio. Problemas como la deforestación.

Bueno ya hay deforestación. De todos modos, bajó la cantidad de hijos por mujer. Pero no es algo planificado, es riqueza porque cada hija después representa una dote. Además, en Timor hay otra cosa muy interesante: el concepto de amistad no existe. Vos no tenés amigos, vos no haces amigos; sos de un clan y tenés otro clan del cual formas parte porque casaste a uno de tus hijos o porque se casó tu hija. Es una sociedad tan pobre que no puede sustentar amistades, porque tal vez a ese amigo no lo puedas alimentar y vos tenés que asegurar la permanencia de tu clan. No se puede perder el tiempo con amigos, de la misma manera que no tienen mascotas. La mascota se come, el perro se come. Cuando fui hace diez años, la idea de mascotas era totalmente insólita para ellos. A lo sumo, la mascota era el gallo de riña.

\section{O sea que no hay bares, no hay lugares donde la gente va a tomar un café con alguien.}

Hay bares para los malae. Los malae son los extranjeros, había mucho bar para ellos. Hay algunos bares para nacionales, pero no es la idea de la amistad. Y otra cosa que no existe en Timor: la queja. Nadie se queja. No está bien visto quejarte; la cultura asiática en general lo condena.

En Argentina, la queja es una forma habitual de encarar lo cotidiano. Esto incluso ante la mirada incrédula de personas que vienen de lugares con problemas "más graves". ¿ $\mathrm{Ni}$ siquiera con todos los problemas que enfrenta Timor se permite la queja?

Nunca, la queja no es bien vista. Vos decís "no, no sabes lo que me pasó, vino y me dijo y yo le dije y después me dijo y yo le dije" o como acá: "esto es un desastre". Eso no está bien en Timor, aunque se estén muriendo de hambre la queja no existe. A mí lo que más me pasaba viviendo en Timor es que volvía a la Argentina y me chocaba la gente quejándose. 
Te estableciste junto con tu familia en Timor en calidad de experta electoral de Naciones Unidas, supervisando la organización de elecciones tras años de conflicto violento. ¿Cómo era el trabajo intelectual y organizativo previo a tu tarea en el campo?

Primero, siempre hay lo que se llama un National Mission Assessment (NAM). Es una misión que va al país y hace una evaluación de la situación electoral. Yo llegué en 2008 para preparar la primera elección municipal de sucos de 2009. Pero mi llegada tuvo que ver con un assessment hecho por la United Nations Electoral Assistance Division (UNEAD). Ellos hacen un informe del que resulta un proyecto a ser homologado por el gobierno. Con la firma del gobierno se obtiene el mandato de la misión y el mandato en tanto misión electoral. No todas las misiones electorales hacen lo mismo. En el caso de Timor para el año 2009, las elecciones las iban a llevar adelante las autoridades nacionales y nosotros íbamos a trabajar como advisers, o sea, como asesores dentro de las instituciones gubernamentales

Después hay un plan del que se parte y tenés un jefe de equipo obviamente. Uno hace propuestas con la contraparte y la contraparte te dice si le gustan o no. Cuando yo llegué, lo que también tenía que hacer era preparar el presupuesto de las elecciones de 2009 para que cubriese todas las campañas de educación pública necesarias. Básicamente, que todos los ciudadanos estuvieran informados y preparados para votar. Entonces, primero que hay que trabajar en la institucionalidad del proceso. Esto lleva a la descentralización, ya que la comisión electoral tenía a su vez sus oficinas y comisarios en cada uno de los quince distritos. Por esto yo trabajaba a nivel local en lugares diferentes como Baucau, Oecusse o Ataúro. Se trabaja en que las personas se inscriban, en poder llegar a los lugares remotos de difícil acceso y que las personas estén empadronadas. Todas cuestiones de la preparación de las elecciones Otra parte del trabajo era hacia el interior de la Comisión Electoral. Preparando a todos los grupos de la sociedad civil para que puedan hacer observación electoral. En eso, la iglesia de Timor tiene un rol muy particular. Las observaciones electorales eran manejadas en gran medida por la iglesia católica.

\section{¿Qué escenario de organizaciones de la sociedad civil encontraste en Timor? ¿Cómo fue trabajar con ellas?}

La sociedad civil es un componente necesario, pero a la vez complicado. Nunca es un componente fácil. Dependiendo del país puede tener diferentes 
perfiles. En el caso de Timor, tenías organizaciones internacionales religiosas dedicadas a cuestiones de maternidad u organizaciones dedicadas a asuntos electorales como International IDEA. Tenés todos los players internacionales que cumplen un rol y no siempre son amigos. A veces son competidores dependiendo de su posición.

Después tenés la sociedad civil local. Era una sociedad civil, en el momento que yo llegué y probablemente esto cambió, incipiente y con muy poquitos años de vida. La mayoría de las organizaciones locales se habían fundado en 2001 y 2002. Algunas de ellas hacían muy buenos trabajos cuando lo hacían en partnership con organizaciones internacionales. Después tenés, como todo, muchas organizaciones que son creadas ad hoc con lindos nombres, lindas ideas, pero están más a la pesca de fondos internacionales. Debo decir que algunas de las organizaciones más efectivas y mejor organizadas eran de la sociedad civil femenina. También eran efectivas las organizaciones dedicadas a cuestiones de acceso al agua e higiene y a temas humanitarios como los desplazados.

A pesar de esto, era difícil porque la mayoría eran muy débiles institucionalmente. Con el asesor del Primer Ministro para la Sociedad Civil, lo que hicimos básicamente fue un assessment de cuáles eran las organizaciones que ya estaban trabajando en cuestiones de educación cívica: en qué partes del país, con qué programas, con qué financiación, en qué idiomas. Entonces organicé un comité al interior de Naciones Unidas de trabajo en educación cívica donde nos juntábamos todas las agencias -UNICEF, UNESCO, UNDP, DPKO, UNFPA, ACNUR-e invitábamos a las organizaciones internacionales. Con esto veíamos quien estaba financiando a qué ONG en lo que respecta a educación cívica.

Esto es muy interesante, en tanto la cuestión de la construcción electoral es cuestión de la construcción de la democracia. Hablar de estas cuestiones con gente local debe ser impresionante porque quizás no pasaron por experiencias democráticas y liberales. ¿Cómo fue hablar de esto con ellos?

Una de las sorpresas que tuve -no solo en Timor sino también en otros países de Asia- es que se trata de una sociedad tribal, entonces hay otra interpretación de lo comunitario. Ya hay una mentalidad que es la mentalidad de la comunidad y tiene toda una impronta. Y lo que tiene el sistema de sucos y aldeias -había 442 sucos y más de mil aldeias en un territorio relativamente pequeño- es que ya hay un entramado del ejercicio del poder civil sin intervención del poder militar, y si bien no es democrático en sí mismo, ya es un ejercicio de civilidad. 
Ahora cuando el sistema democrático se imprime sobre Timor lo hace sobre ese sistema, integrando los sucos y las aldeias. Entonces cuando son las elecciones municipales de sucos y aldeias, que son cargos honoríficos, genera un choque con la municipalidad que sí tiene presupuesto. Entonces hay roces entre los dos niveles de gobernabilidad.

Respecto al tema de la mentalidad, yo diría que me sorprendió un poco lo sofisticado del pensamiento político y la comprensión de la importancia de la participación y la toma de decisiones a nivel local, porque realmente las mentalidades tribales - por lo menos las que yo conozco- tienen esa impronta muy fuerte de culturas locales con redes de ejercicio del poder civil. Por supuesto, cómo se eligen los grupos de poder dentro de las tribus y las aldeias no es lo mismo que en la democracia representativa. Lo que quiero decir es que ya hay cierto espíritu cívico que es interesante.

\section{¿Algo que quizás nos hace pensar más en una democracia directa que en una democracia republicana?}

Totalmente, tienen algo de democracias directas. Ellos no lo llamarían así, pero era una democracia directa en un sentido muy puro te diría porque no es democracia directa impuesta.

\section{Por lo que decís, pareciera que ya había cierto lenguaje de participación cívica y tenías que traducirlo a un sistema electoral. ¿Cuáles fueron los desafíos de a la educación cívica?}

Los desafíos tienen que ver con la geografía de Timor, tanto la geográfica física como la geografía cultural. Por ejemplo, el problema de las diferencias de género que son muy atávicas. Es una sociedad machista y tribal donde la educación es percibida como algo un poco ajeno a la cultura de la comunidad, donde se necesita ante todo que la gente sea mano de obra para la agricultura en una economía de subsistencia. Además, otro problema es la cultura del trabajo: trabaja el que tiene menor escala social. El chefe no trabaja porque un gran jefe no va a trabajar. De hecho, en las familias, el hermano mayor trabaja mucho menos porque el menor trabaja para él. La comida se reparte de la misma manera. Primero come el marido después el hijo más grande y así hasta el más chiquito. Todas estas cuestiones de supervivencia afectan obviamente la educación cívica. El gran desafío es cómo acercar la cultura cívica a la vida de todos los días cuando ésta es tan ruda, cuando la preocupación es si tenés o no tenés arroz, si tenés agua, si en el parto tuviste al bebé o no. 
A esto se suma la cuestión de género. Las reuniones de suco y las de aldeia se hacen en un espacio común donde solo están los hombres. Entonces hay muchos desafíos para superar y cosas por explicar: la necesidad de participar políticamente, de informarse, de saber quiénes van a ser los candidatos, de saber que te podés candidatear, de saber que no tenés por qué votar a alguien si su propuesta no te interesa, de saber que necesitás tener tu tarjeta de identificación, etc. También está el analfabetismo y la pluralidad lingüística. No todo el mundo habla lo mismo, se habla bahasa, tetum, variantes del tetum y otras lenguas. Entonces, teníamos que trabajar con las radios locales, ya que en aquella época el celular no estaba en todos lados. Todos estos son desafíos en un país muy pobre y con muy pocos recursos. ¡Y muy aislado, muy aislado! Aislado del mundo por su geografía y su pasado como colonia de Indonesia.

Por otra parte, están las cuestiones políticas al interior de la Comisión Electoral. Los intereses de las quince personas que eran los comisarios y que por suerte ahora son siete. Por lo general, las comisiones electorales son bastante ineficientes, pero son necesarias en situaciones de posconflicto. Porque la comisión electoral refleja un acuerdo de paz en un país luego de una situación de conflicto. Estas comisiones con tanta gente, a diferencia de países con comisiones más sofisticadas, representan ese acuerdo.

Otro gran desafío es la corrupción. Que los fondos recibidos de la comunidad internacional sean bien gastados. Esto afecta porque los fondos destinados a educación cívica o a tener un manual o charlas pueden irse al caño. Obviamente lo primero que se recorta son los fondos para educación cívica.

¿Qué desafíos tiene la estructuración de una oferta electoral comprensible y accesible para culturas tribales como las de Timor? En Sudáfrica tuvieron que poner fotos en lugar nombres de candidatos y en Brasil números porque había una cultura vinculada a la lotería de quiniela. ¿Cuál fue la vuelta que le encontraron en Timor para engarzar una oferta electoral comprensible con el universo simbólico de la cultura local?

En Timor es muy fácil. A través de los jefes de sucos y los jefes de aldeias llegás a toda la población. Si el jefe es quien te explica cómo votar -era fácil, con boletas sencillas-, el desafío no era el acto de votar, sino que todos estuvieran informados. Es una sociedad que no tiene acceso a grandes medios de comunicación y que vive muy aislada, porque en Timor las distancias son cortas, pero nadie tiene auto y las carreteras eran pésimas. Transporte común casi no existía. 
La estructura electoral más importante era la Iglesia, los sucos y las aldeias. Aquí se daba una interrelación entre estas estructuras y los órganos electorales del gobierno. Por ejemplo, el jefe del Secretariado Técnico Da Administração Eleitoral (STAE) era también un jefe religioso. En Timor, las elecciones no son organizadas por la Comisión Electoral sino por el STAE. Es un organismo técnico que depende del Ministerio del Interior y del Primer Ministro. En cambio, la Comisión Electoral era independiente y estaba bajo la órbita del presidente. Entonces la comisión podía hacer un seguimiento técnico del STAE con independencia.

Entonces el jefe del STAE era también jefe religioso y como tal tenía un nivel social dentro la lógica tribal muy importante. Él iba a las aldeias y los sucos y hacía reuniones con los jefes para lograr consensos sobre la participación electoral a través de las reglas consuetudinarias de gobierno tradicional. Eso era muy útil en Timor.

Hablar de democracia moderna es hablar de partidos políticos. Mencionabas el rol de los jefes en el proceso electoral a nivel local en los sucos y aldeias. ¿Qué llegada tenían los partidos políticos a esas localidades? ¿Partidos como FRETILIN o CNRT tenían presencia en esos territorios?

Yo en este momento no lo recuerdo. Lo que recuerdo es que el FRETILIN, obviamente, era súper popular. Y había como quince o veinte partidos políticos que eran grupúsculos que andaban dando vueltas por ahí. Pero no, ellos se apoyaban sobre lo que ya estaba. Obviamente que el FRETILIN era muy popular, realmente tenía un alto grado de aceptación en todo el país. Xanana era muy popular, Ramos Horta era muy popular en ese momento.

Llegas a Timor como experta electoral para una misión de paz de Cascos Azules. Para una misión integrada con fuerzas armadas internacionales con el objetivo de poner fin a la violencia desatada en 2006 y fortalecer al gobierno civil. Ya desde la retirada de las fuerzas indonesias luego del referéndum, la vida política de Timor se vio interrumpida por la violencia. ¿Las elecciones también fueron violentas?

Las dos elecciones que yo viví en Timor no fueron elecciones a las que yo catalogaría como violentas o en donde primó la violencia como parte del conflicto electoral. De hecho, en las elecciones 2012 hicimos el "Pacto de Eleição Pacífica" y donde estuvieron todos los partidos políticos en todas las 
reuniones. No quiere decir que no hubiera violencia en la sociedad, me refiero a que no fueron necesariamente campañas electorales donde murieron candidatos, o donde hubiese atentados en los actos de campaña o donde las autoridades electorales eran atacadas, o donde los centros de los partidos políticos también fuesen atacados, o donde hubiese un enorme vandalismo de los materiales de campaña, o donde los centros electorales fueran atacados. No había un clima de violencia política en torno a lo que era el proceso electoral. Ahora, desde ya no era una sociedad pacífica. De hecho, a la noche la ciudad era patrullada y había toque de queda cuando yo llegué. Habían prendido fuego a media ciudad.

Respecto a esto que estás diciendo parece haber, a nivel de la élite política, de la élite dirigencial de todos los niveles, como un acuerdo en que estaba bien hacer las elecciones ¿Eso se vinculaba con la Independencia de algún modo?

Desde una mirada personal, porque como te digo, yo no estaba en la rosca política, yo creo que sí. Tal vez los de Polítical Affairs lo tienen más claro. Me parece que, a nivel de la clase política, y te lo digo yo que trabajé con Xanana, del lado de la elites había un consenso más allá de las diferencias ideológicas. Se sentían cómodos con la idea de la continuidad política, con la continuidad democrática. No veo dónde pudiera estar la conveniencia para ellos, que estaban en puestos ya de poder, de desestabilizar la democracia; no era necesario. Podían simplemente llegar a pactos, y en ese sentido me parece que la oposición al FRETILIN estaba también interesada en llegar a pactos. Pero esto es mi opinión personal, cuando yo estuve allá no vi intención de desestabilizar, ellos comenzaban a sentir los beneficios de la estabilidad, y sobre todo los beneficios de ser la élite gobernante.

\section{¿Cómo fue su experiencia con Xanana Gusmão? ¿Cómo lo describiría?}

No se puede negar que él fue una figura enormemente carismática. Ahora, ¿como primer ministro? No sé, era un líder, con un liderazgo más informal. Que vos puedas llevar adelante una resistencia armada no quiere decir que después te den un gobierno y sepas qué hacer con eso; son cosas distintas. Después tomaron decisiones por cuestiones nacionalistas con las que yo estaba profundamente en desacuerdo. Por ejemplo, una decisión que a mí me pareció muy equivocada fue la decisión de que no se enseñara más en lengua bahasa, y el tetun es un idioma muy limitado. Le falta vocabulario porque justamente es un idioma que está vinculado con la supervivencia. Entonces hicieron del 
portugués y del tetun lenguas nacionales, y la educación pasó a ser en portugués y tetun de un día para el otro. Entonces, ¿qué pasó? Los que hablaban bahasa, no hablaban ni tetun ni portugués y los que sí hablaban tetun lo hacían en sus casas.

¿Sentiste la presencia, de alguna forma, de Indonesia tratando de inmiscuirse en el proceso?

No, lo que sentí muy fuertemente fue la presencia de Australia y de China más que Indonesia. En ese momento yo creo que las relaciones con Indonesia estaban muy enfriadas, incluso amistosas en términos generales. Pero, lo que sentí fue una enorme presencia de China y de Australia.

\section{¿Y personificadas en qué? ¿En prensa, pensiones?}

No, en dádivas al gobierno. El ministerio de defensa fue construido con donaciones, como un regalo de China. O sea, todos los ministerios los construía China como un regalo. No sé qué pedían a cambio. Y después, yo me enteraba de mucha presión sobre todo con el problema del agua. China quería construir represas en el país. Por otra parte, estaba el petróleo. Ahí veías lo que era el tema de El Fondo del Petróleo del lado también de Australia y Estados Unidos.

\section{¿Te parece que desde América Latina hay alguna lección que podamos aprender de Timor? ¿Errores y aciertos que debiéramos estar mirando para mejorar en América Latina o Argentina?}

Algo que personalmente me impactó y que después lo volví a ver repetido en muchos lugares es que cuando uno se enfrenta a situaciones en donde están estos problemas de posconflicto hay que saber que hay potencias en juego. Nunca tenés conflictos de este tipo sin tener fuerzas extranjeras que también están interesadas en que las cosas se muevan para un lado o para el otro.

\section{Muchas gracias, Albertina. No es frecuente hablar sobre Timor con personas con experiencia directa. Eso es muy valioso.}

Gracias a ustedes. No saben lo lindo que es poder hablar con gente a la que le interesa Timor. Porque una de las grandes frustraciones que yo tenía era que a nadie le importaba Timor. Cuando yo volvía a Buenos Aires para ver a mi 
familia me preguntaban "¿Y qué tal allá?". A la gente le cuesta tener empatía con lo que no pueden imaginar. Es un país desconocido pero maravilloso.

Cierro con una anécdota. Desde que pasé por Timor me encantan los cocodrilos. Aparecían en la playa: cocodrilos de rio, de manglar y de mar. Y para ellos, que son animistas, los cocodrilos son los antepasados, entonces no se los mata porque vos no matas a tus antepasados, quedás maldito si hacés eso. Pero si te come un cocodrilo es tu culpa, quiere decir que vos no eras muy buena persona. Si el cocodrilo te come es porque había algo mal con vos, sino el cocodrilo no te va a comer porque es tu ancestro. Entonces recuerdo que una vez cuando estaba yendo a la oficina del PNUD que daba a la comisión electoral estaba justo frente a la playa. De repente veo que por la costanera hay como un malecón y está toda la gente así asomada mirando un cocodrilo enorme pero tranquilo. Nadie gritando ni tirando piedras ni nada en pleno centro de la ciudad. Y son esas anécdotas que personalmente a mí me marcan tanto porque es en lo cotidiano del día a día donde ves esas diferencias. No son diferencias irreconciliables, pero te abren la cabeza. 

